\title{
The origin of galactic cosmic rays *
}

\author{
Jörg R. Hörandel \\ Radboud University Nijmegen, Department of Astrophysics, P.O. Box 9010, 6500 GL Nijmegen, The Netherlands
}

\begin{abstract}
The origin of galactic cosmic rays is one of the most interesting unsolved problems in astroparticle physics. Experimentally, the problem is attacked by a multi-disciplinary effort, namely by direct measurements of cosmic rays above the atmosphere, by air shower observations, and by the detection of $\mathrm{TeV} \gamma$ rays. Recent experimental results are presented and their implications on the contemporary understanding of the origin of galactic cosmic rays are discussed.
\end{abstract}

Key words: cosmic rays, origin, acceleration, propagation, energy spectra, mass composition, extensive air showers PACS: 96.50.S-, 96.50.sb, 96.50.sd, 98.70.Sa

\section{Introduction}

The Earth is permanently exposed to a vast flux of highly energetic particles, fully ionized atomic nuclei from outer space. The extraterrestrial origin of these particles has been demonstrated by V. Hess in 1912 [1] and he named the particles "Höhenstrahlung" or "Ultrastrahlung". In 1925 R. Millikan coined the term "Cosmic Rays". They have a threefold origin. Particles with energies below $100 \mathrm{MeV}$ originate from the Sun. Cosmic rays in narrower sense are particles with energies from the $100 \mathrm{MeV}$ domain up to energies beyond $10^{20} \mathrm{eV}$. Up to several $10 \mathrm{GeV}$ the flux of the particles observed is modulated by the 11year cycle of the heliospheric magnetic fields. Particles with energies below $10^{17}$ to $10^{18} \mathrm{eV}$ are usually considered to be of galactic origin. A proton with an energy of $10^{18} \mathrm{eV}$ has a Larmor radius $r_{L}=360 \mathrm{pc}$ in the galactic magnetic field $(B \approx 3 \mu \mathrm{G})$. This radius is comparable to the thickness of the galactic disc and illustrates that particles at the highest en-

\footnotetext{
ऋ Invited talk given at the Roma International Conference on Astro-Particle physics (RICAP07) June 20th - 22nd, 2007.

URL: hoerandel.com (Jörg R. Hörandel).
}

ergies can not be magnetically bound to the Galaxy. Hence, they are considered of extragalactic origin.

The energy density can be inferred from the measured differential energy spectrum $d N / d E$ [2]

$\rho_{E}=\frac{4 \pi}{c} \int \frac{E}{\beta} \frac{d N}{d E} d E$,

where $\beta c$ is the velocity of particles with energy $E$. For galactic cosmic rays the major contribution to the total energy density originates from particles with energies around $1 \mathrm{GeV}$. Such particles are strongly influenced by the heliospheric magnetic fields. Outside the heliosphere, in the local interstellar environment an energy density $\rho_{E}^{L I S}=$ $1.1 \mathrm{eV} / \mathrm{cm}^{3}$ is obtained (Ref. 3] p. 12). The parameterization of the measured galactic cosmic-ray flux according to the poly-gonato model 4 results in a density $\rho_{E}^{g a l}=0.43 \mathrm{eV} / \mathrm{cm}^{3}$. This is the measurable energy density at Earth (for an average modulation parameter $M=750 \mathrm{MeV}$, see (1) in Ref. (4). This implies that less than half of the energy flux can be registered directly at Earth.

In this article we will give an overview on recent experimental results and their implications on the contemporary understanding of the origin of galactic cosmic rays. As space is limited here, the reader may 
also consider further recent reviews by the author 4156778 .

Progress in the understanding of the origin of galactic cosmic rays emerged mainly from observations in three complementary disciplines. The direct measurement of cosmic-ray particles above the atmosphere in outer space and on stratospheric balloons (Sect.22). At energies exceeding $10^{15} \mathrm{eV}$ the steeply falling cosmic-ray energy spectrum requires experiments with large detection areas exposed for long times, at present, only realized in ground based installations (Sect. (4). With such detectors extensive air showers are detected, which originate in interactions of high-energy particles in the atmosphere (Sect. 31). And, finally, the observation of $\mathrm{TeV} \gamma$-rays (Sect.51).

\section{Direct measurements}

Detectors above the atmosphere allow a precise determination of the composition of cosmic rays and the measurement of energy spectra for individual elements, presently, up to about $10^{14} \mathrm{eV}$. These findings are very important to understand the propagation of the particles in the Galaxy.

In the $100 \mathrm{MeV}$ energy domain detailed studies of the isotopic composition of cosmic rays and the comparison to the local galactic abundance indicates that cosmic rays are accelerated out of a sample of well mixed interstellar matter [9]. The propagation time of the particles in the Galaxy has been determined to be $\tau_{\text {esc }}=(15.6 \pm 1.6) \cdot 10^{6} \mathrm{yr}[1011112$. At $\mathrm{GeV}$ energies the elemental composition has been studied in detail, finding all elements of the periodic table in cosmic rays, see e.g. 6]. Some elements, as e.g. boron are produced during the propagation in the Galaxy through spallation of heavier elements such as carbon. Investigations of the abundance ratios of different elements (like the boron-to-carbon ratio) yield the column density of matter traversed by the particles in the Galaxy [10]13.

At present, several groups try to extend the direct measurements to higher energies towards the knee (at about $4.5 \cdot 10^{15} \mathrm{eV}$ ) in the all-particle spectrum, benefiting from long-duration balloon flights 15 . Among these are the ATIC [16, CREAM [17, and TRACER [18, experiments. The latter is presently with an aperture of $5 \mathrm{~m}^{2}$ sr the largest cosmic-ray detector for direct measurements. It comprises several electromagnetic detectors, namely an acrylic Čerenkov counter, a proportional tube array, and a

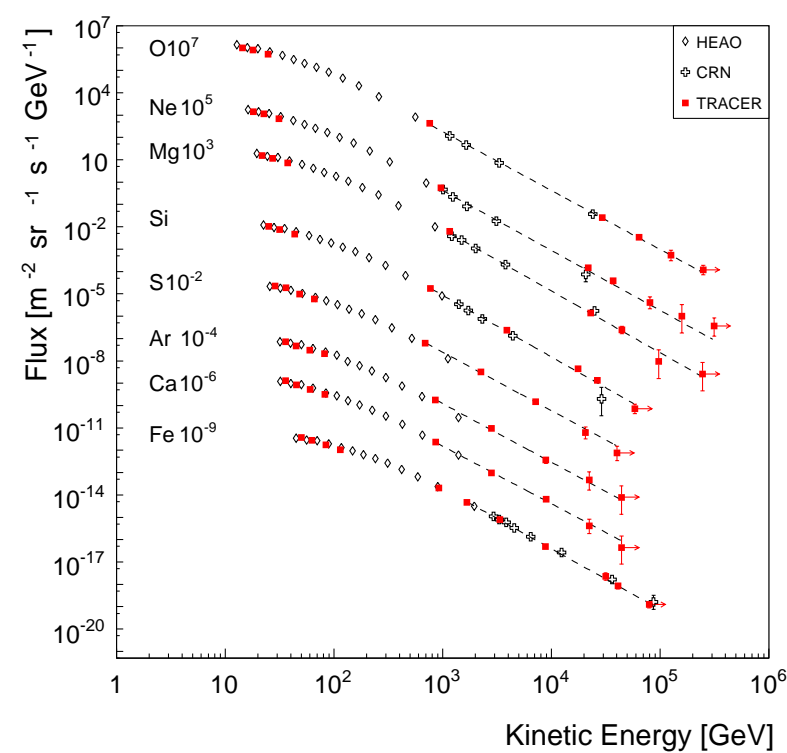

Fig. 1. Energy spectra for individual elements as measured by the TRACER, HEAO, and CRN experiments 14.

transition radiation detector, thus covering a large energy range from $10 \mathrm{GeV} / \mathrm{n}$ up to $10^{5} \mathrm{GeV} / \mathrm{n}$ with single-element resolution. Spectra for elements from oxygen to iron as measured by TRACER during an Antarctic circumpolar flight in 2003 are compiled in Fig.114]. The data extend previous results from the space experiments HEAO (satellite) and CRN (on the Space Shuttle) to higher energies. Currently, the data of a flight from Sweden to Alaska in 2006 are analyzed. TRACER has been flown with an upgraded electronics being able to record elements from boron to iron. The new data are expected to extend measurements of the boron-to-carbon ratio up to $10^{5} \mathrm{GeV} / \mathrm{n}$, thus, providing, unprecedented information of the propagation properties approaching the knee in the energy spectrum.

Frequently, the question arises whether the energy spectra of protons and helium have the same spectral index. Figure2 shows a compilation of data, for details and references see Ref. [6]. From the figure it seems to be clear that the helium spectrum is indeed flatter as compared to protons. Due to spallation of nuclei during their propagation and the dependence of the interaction cross section $\propto A^{2 / 3}$ one would expect a slightly flatter spectrum for helium nuclei as compared to protons. Following [19] the difference should be of the order of $\Delta \gamma \approx 0.02$. However, fits to the experimental data (lines in Fig.2) yield $\gamma_{p}=$ $-2.71 \pm 0.02$ and $\gamma_{\mathrm{He}}=-2.64 \pm 0.02$ [4, resulting in a difference $\Delta \gamma=0.07$. 


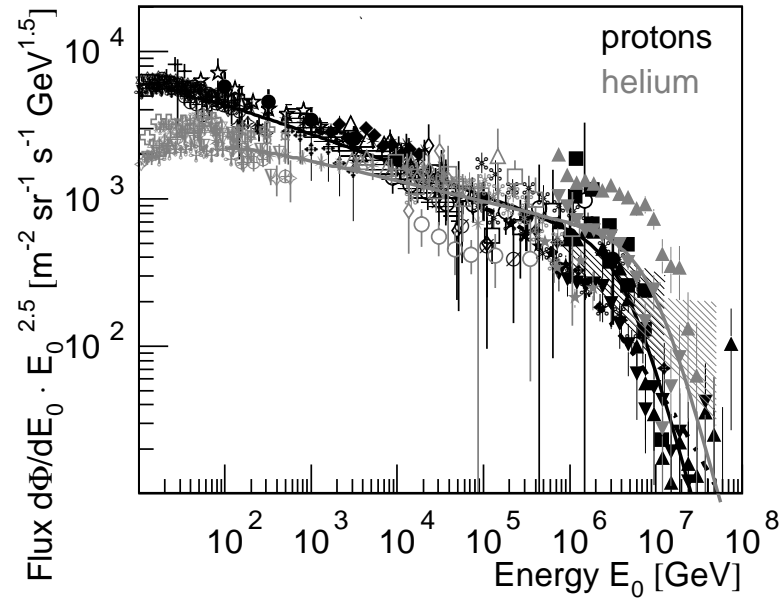

Fig. 2. Energy spectra for protons (black) and helium nuclei (grey). For a list of experiments and references see Ref. 6]. The lines represent fits according to the poly-gonato model [4.

\section{Extensive air showers}

When high-energy cosmic-ray particles penetrate the Earths atmosphere they interact and generate a cascade of secondary particles, the extensive air showers. Two types of experiments may be distinguished to register air showers: installations measuring the longitudinal development of showers (or the depth of the shower maximum) in the atmosphere by observations of Čerenkov or fluorescence light and apparatus measuring the density (and energy) of secondary particles (electrons, muons, hadrons) at ground level.

The shower energy is proportional to the total light collected or to the total number of particles recorded at observation level. More challenging is an estimation of the mass of the primary particle. It is either derived by a measurement of the depth of the shower maximum $X_{\max }$ and the fact that the depth of the shower maximum for a primary particle with mass $A$ relates to the depth of the maximum for proton induced showers as

$X_{\max }^{A}=X_{\max }^{p}-X_{0} \ln A$,

where $X_{0}=36.7 \mathrm{~g} / \mathrm{cm}^{2}$ is the radiation length in air 2021]. Or, measuring the electron-to-muon ratio in showers. A Heitler model of hadronic showers [21] yields the relation

$\lg \left(N_{e} / N_{\mu}\right)=C-0.065 \ln A$.

This illustrates the sensitivity of air shower experiments to $\ln A$. To measure the composition with a



Fig. 3. Number of electrons vs. number of muons at shower maximum for fully simulated showers (symbols). The lines represent predictions of a Heitler model: solid - constant mass for protons and iron nuclei (4), dashed - constant energy (5), and dotted - constant depth of the shower maxi$\operatorname{mum} X_{\max }$ [6].

resolution of 1 unit in $\ln A$ the shower maximum has to be measured to an accuracy of about $37 \mathrm{~g} / \mathrm{cm}^{2}$ (see (2)) or the $N_{e} / N_{\mu}$ ratio has to be determined with an relative error around $16 \%$ (see (3)). Due to the large intrinsic fluctuations in air showers, with existing experiments at most groups of elements can be reconstructed with $\Delta \ln A \approx 0.8-1$.

The detection principle is illustrated in Fig. 3 , depicting the number of electrons as function of the number of muons at shower maximum. The symbols represent fully simulated showers with discrete energies in steps of half a decade, for details see Ref. 21]. The lines represent predictions of a Heitler model of hadronic air showers 21. The solid lines are lines of constant mass

$$
\left.N_{e}\right|_{A}=7.24 \cdot A^{-0.16} N_{\mu}^{1.16}
$$

for primary protons and iron nuclei. The dashed lines correspond to a constant energy, following

$$
\left.N_{e}\right|_{E_{0}}=5.30 \cdot 10^{7}\left(E_{0} / \mathrm{PeV}\right)^{1.37} N_{\mu}^{-0.46} .
$$

The sets of lines define a parallelogram giving the axes for energy and mass in the $N_{e}-N_{\mu}$ plane as indicated by the arrows. The dotted lines represent a constant $X_{\max }$, separated by $100 \mathrm{~g} / \mathrm{cm}^{2}$ according to

$$
\left.\lg N_{e}\right|_{X_{\max }}=\frac{X_{\max }+287.9 \mathrm{~g} / \mathrm{cm}^{2}}{569.6 \mathrm{~g} / \mathrm{cm}^{2}}+1.02 \lg N_{\mu} .(6)
$$




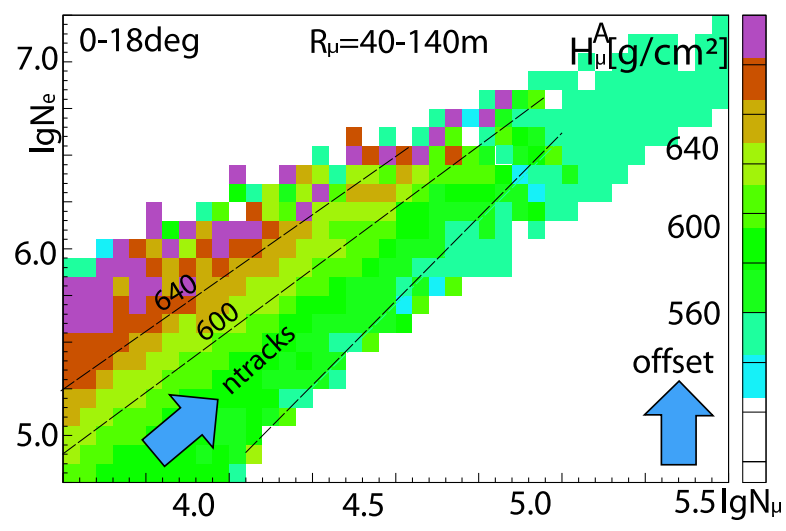

Fig. 4. Number of electrons vs. number of muons for showers measured with the KASCADE experiment, the third dimension is the muon production hight [22].

A similar plot is presented in Fig. 4, showing the $N_{e}-N_{\mu}$ plane for showers measured with the KASCADE experiment. The third dimension gives the production hight of muons 22. In the $N_{e}-N_{\mu}$ plane light primary elements are expected in the upper left part of the figure. This is underlined by the measurements yielding in this area deeply penetrating showers. Attention should be payed when the two figures are compared: $X_{\max }$ for the electromagnetic component (Fig. 3) is compared to $X_{\max }$ for the muonic component $\left(E_{\mu}>0.8 \mathrm{GeV}\right.$, Fig.4). Fig.3 represents $N_{e}$ and $N_{\mu}$ at the shower maximum, Fig.4 gives values measured at ground. For muons the lateral distributions are integrated in the range interval from 40 to $200 \mathrm{~m}$ only in Fig. 4 .

By detecting two quantities (e.g. $N_{e}$ and $N_{\mu}$ or $N_{e}$ and $X_{\max }$ ), energy and mass of the primary particles can be deduced. The arrival direction of cosmic rays is determined by measurements of the arrival times of secondary particles at the detectors.

\section{Indirect measurements}

Air shower experiments extend the findings of direct measurements to highest energies. They provide information on the all-particle energy spectrum, the (average) mass composition, and, recently, information on energy spectra for elemental groups. These results contribute to the understanding of the origin of structures in the all-particle spectrum, like the knee or the second knee.

The all-particle energy spectrum up to about $10^{18} \mathrm{eV}$ is reasonably well known. The spectra obtained by different experiments agree well in shape and absolute flux taking a $\pm 10 \%$ uncertainty in the

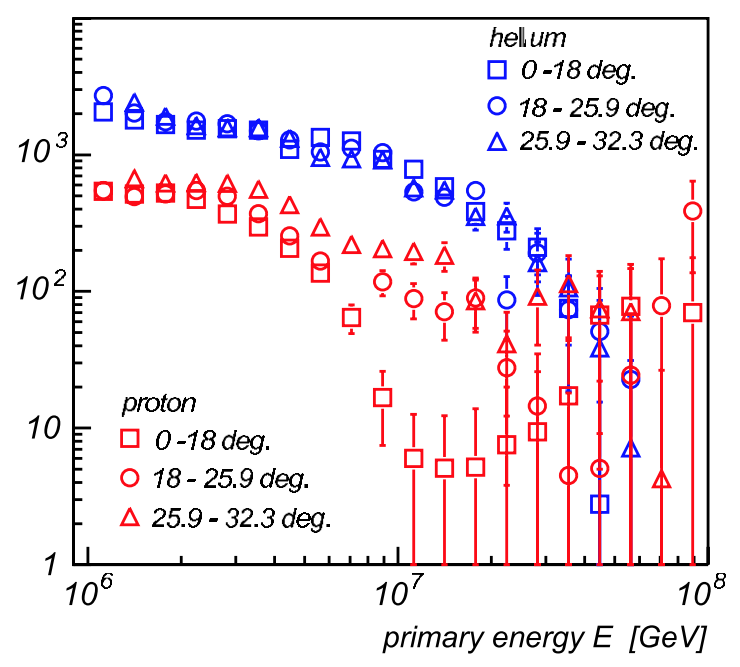

Fig. 5. Energy spectra for primary protons and helium nuclei, unfolded from air showers registered by KASCADE within three zenith angle intervals [24].

energy determination of the individual experiments into account 4 . The spectrum can be described by a power law $d N / d E \propto E^{\gamma}$, with an spectral index changing at the knee $\left(E_{k}=4.5 \mathrm{PeV}\right)$ from $\gamma=-2.7$ to $\gamma=-3.1$.

For the mean (logarithmic) mass of cosmic rays there seems to be a dependence on the technique applied for the air shower observations [23]. Two classes of experiments may be distinguished: detectors measuring the depth of the shower maximum and experiments measuring (the lateral distribution of) particles at ground level. This indicates inconsistencies in the interaction models used to interpret the data. For example, introducing lower inelastic cross sections in the interaction model QGSJET 01 and slightly increasing the elasticity, good agreement between the two classes of experiments can be achieved 23. Interpreting the data with this model yields a consistent picture with a mean logarithmic mass smoothly continued from direct measurements up to about $10^{15} \mathrm{eV}$, then exhibiting an increase up to energies around $10^{17} \mathrm{eV}$.

A big step forward in understanding the origin of the knee in the all-particle energy spectrum are the results of the KASCADE experiment. The latter comprises a $200 \times 200 \mathrm{~m}^{2}$ scintillator array to register the electromagnetic and muonic component 25, a hadron calorimeter equipped with liquid ionization chambers 26, and a limited-streamer tube muon tracking detector [27]. The simultaneous detection of different shower components allows detailed studies of hadronic interaction models 


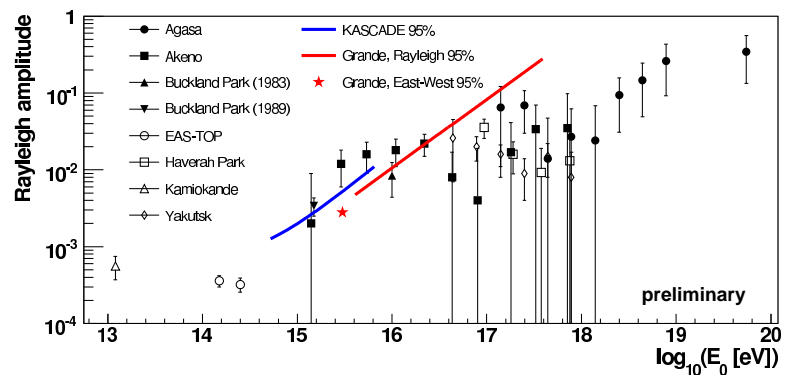

Fig. 6. Rayleigh amplitudes as function of energy as observed by different experiments [39].

$282930 \mid 3132$ 33 34. The most consistent models are then applied to unfold energy spectra for five groups of elements from the measured air shower data [35. The energy spectra of the light elements (protons and helium) exhibit a depression of the flux at high energies while the spectra of the heavier groups continue to follow power laws to higher energies. The results obtained using different hadronic interaction models to interpret the data agree qualitatively. However, there are differences in the absolute flux obtained, indicating inconsistencies in the interaction models. These results have recently been confirmed by an analysis taking also inclined showers into account 24]. As example, the spectra obtained for primary protons and helium nuclei for three different intervals of the cosmic-ray arrival direction are shown in Fig. 5 , The different data sets are consistent with each other.

Similar energy spectra for groups of elements have been obtained by the EAS-TOP 36, GRAPES-3 37, and Tibet 38 experiments 6]. Overall, a consistent 'standard picture' evolves [4]6] the energy spectra as obtained by direct measurements can be extrapolated to high energies, assuming power laws. The data are compatible with cut-offs in the spectra proportional to their nuclear charge $Z$. The allparticle spectrum and mean logarithmic mass thus obtained are compatible with air shower data. The combined energy spectra for groups of elements obtained from direct and indirect measurements extend over seven orders of magnitude in energy. They are compatible with contemporary models of the acceleration of cosmic rays in supernova remnants and models of the diffusive propagation of the particles in the Galaxy [].

Another important aspect is the anisotropy of the arrival direction of cosmic rays, giving hints to understand the propagation of the particles in the Galaxy. If the knee is caused by leakage of particles from the Galaxy, one would expect less particles arriving from directions perpendicular to the galactic disk. Frequently, the Rayleigh formalism is applied to quantify the observations 40 . Upper limits as obtained by KASCADE and preliminary results obtained by the KASCADE-Grande experiment are compiled together with results from many experiments in Fig.6 [39. An increase of the anisotropy amplitudes can be recognized. However, an interpretation has to take into account that the available statistics decreases roughly as $\propto E^{-3}$, therefore, an increase of the observed anisotropy is expected, which is of the order of the observed increase. The observed anisotropy amplitude is compatible with a diffusion model of the particle propagation 404142 .

\section{TeV $\gamma$-ray astronomy}

Observation of $\mathrm{TeV} \gamma$-rays with atmospheric Čerenkov telescopes, like the HESS 44, MAGIC 45, or VERITAS 46] experiments, give the most direct hints towards the acceleration sites of cosmic rays.

The energy density of galactic cosmic rays amounts to about $\rho_{E} \approx 1 \mathrm{eV} / \mathrm{cm}^{3}$. The power required to sustain a constant cosmic-ray intensity can be estimated as $L_{c r}=\rho_{c r} V / \tau_{e s c} \approx 10^{41} \mathrm{erg} / \mathrm{s}$, where $\tau_{\text {esc }}$ is the residence time of cosmic rays in a volume $V$ (the Galaxy, or the galactic halo). With a rate of about three supernovae per century in a typical Galaxy the energy required could be provided by a small fraction $(\approx 10 \%)$ of the kinetic energy released in supernova explosions. This has been realized already by Baade and Zwicky [47. The actual mechanism of acceleration remained mysterious until Fermi 48 proposed a process that involved interaction of particles with large-scale magnetic fields in the Galaxy. Eventually, this lead to the currently accepted model of cosmic-ray acceleration by a first-order Fermi mechanism that operates in strong shock fronts which are powered by the explosions and propagate from the supernova remnant (SNR) into the interstellar medium [4950|51/52].

The theory of acceleration of (hadronic) cosmic rays at shock fronts in supernova remnants is strongly supported by recent measurements of the HESS experiment [53/43, observing $\mathrm{TeV} \gamma$ rays from the shell type supernova remnant $\mathrm{RX}$ J1713.7-3946, originating from a core collapse supernova of type II/Ib. For the first time, a SNR could be spatially resolved in $\gamma$-rays and spectra 

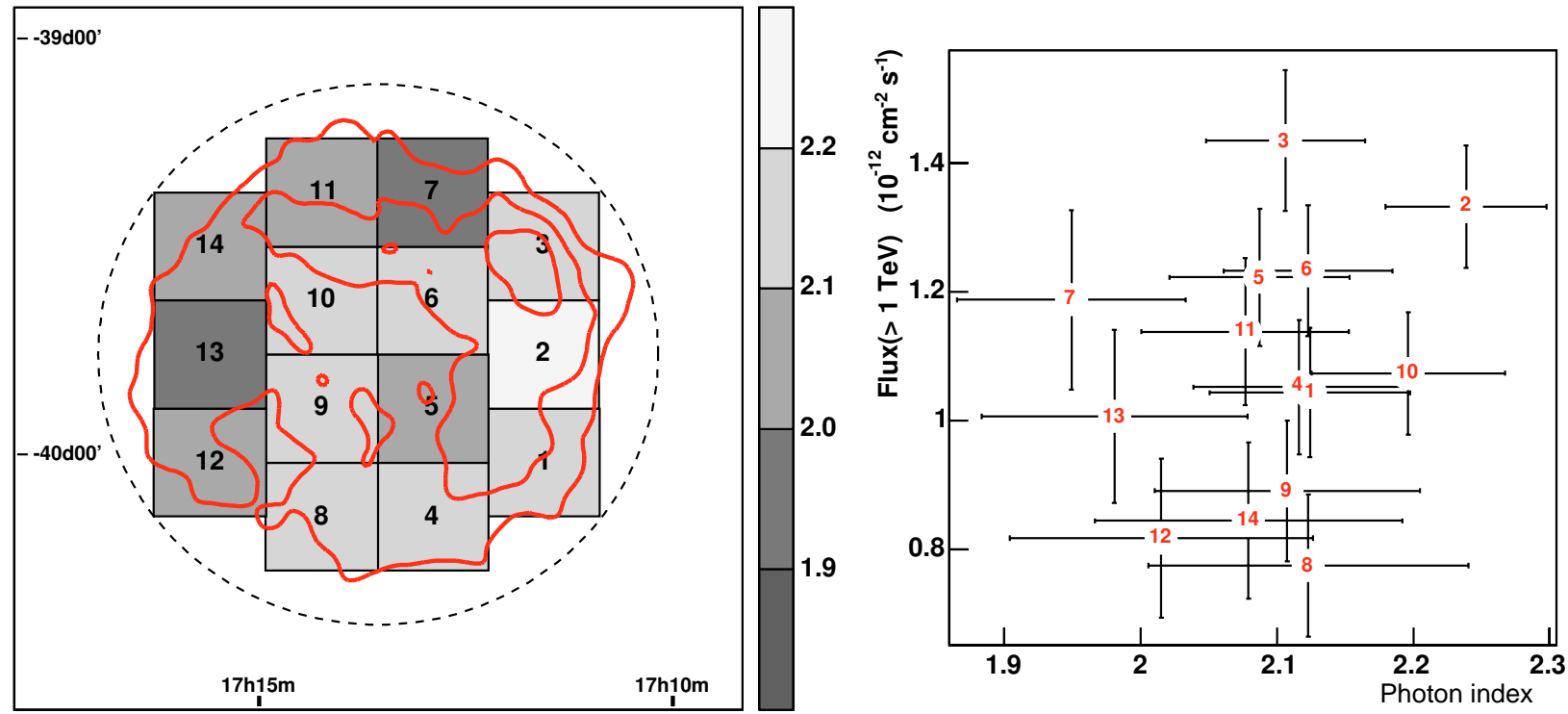

Fig. 7. The SNR RX J1713.7-3946 as seen by the HESS experiment, spatially resolved in TeV $\gamma$-ray light. Left: $\gamma$-ray excess contours, superimposed are 14 fields for which spectra have been obtained independently. The corresponding integral flux is displayed as function of photon spectral index (right). For details see [43].

have been derived directly at a potential cosmic-ray source as can be seen in Fig. 7. The figure shows on the left-hand side excess contours of $\mathrm{TeV} \gamma$-rays. The SNR has been divided into 14 fields for which spectra have been obtained independently [43. The corresponding integral flux values are plotted against the spectral index on the right-hand panel. The measurements yield a spectral index $\gamma=-2.19 \pm 0.09 \pm 0.15$ for the observed $\gamma$-ray flux averaged over the complete source.

A model for the acceleration of hadronic particles in the SNR is supported by measurements in various energy ranges from radio wavelengths to $\mathrm{TeV} \gamma$ rays 54. An important feature of the model is that efficient production of nuclear cosmic rays leads to strong modifications of the shock with large downstream magnetic fields $(B \approx 100 \mu \mathrm{G})$. Due to this field amplification the electrons are accelerated to lower maximum energy and for the same radio/xray flux less electrons are needed. Consequently, the inverse Compton and Bremsstrahlung fluxes are relatively low only. The model predicts that the spectrum of decaying neutral pions, generated in interactions of accelerated hadrons with material in the vicinity of the source, is clearly dominant over electromagnetic emission generated by inverse Compton effect and non-thermal Bremsstrahlung. The measurements are compatible with a nonlinear kinetic theory of cosmic-ray acceleration in supernova rem- nants and imply that this supernova remnant is an effective source of nuclear cosmic rays, where about $10 \%$ of the mechanical explosion energy are converted into nuclear cosmic rays 5455.

Further quantitative evidence for the acceleration of hadrons in supernova remnants is provided by measurements of the HEGRA experiment 56] of $\mathrm{TeV} \gamma$-rays from the SNR Cassiopeia A 57. and by measurements of the HESS experiment from the SNR "Vela Junior" (SNR RX J0852.0-4622) [58.

For completeness, it should also be mentioned that the detection of $\gamma$-rays with $\mathrm{GeV}$ energies contributed to the understanding of cosmic-ray propagation. The diffuse $\gamma$-ray background detected with the EGRET satellite experiment 59 exhibits a structure in the $\mathrm{GeV}$ region, which is interpreted as indication for the interaction of propagating cosmic rays with interstellar matter 60. This is the most direct evidence for cosmic rays propagating in the galactic halo, well outside the galactic disc.

\section{Discussion and outlook}

In the last decade our understanding of the origin of galactic cosmic rays has been significantly improved by multidisciplinary efforts, combining key observations of the direct and indirect measurements of cosmic rays as well as the detection of $\gamma$-rays. The observations by the HESS $\gamma$-ray 
telescope give clear hints that hadronic particles are accelerated in SNR. The data are compatible with a model of first order Fermi acceleration at strong shock fronts. The particles propagate in a diffusive process through the Galaxy. Parameters of the propagation models have been constraint by direct measurements above the atmosphere. The KASCADE experiment has shown that the energy spectra of the light elements exhibit a cut-off structure, while the spectra of heavier elemental groups follow power laws to higher energies. The observed spectra seem to be compatible with the assumption of power laws and a cut-off energy proportional to the nuclear charge. This implies that the knee in the all-particle energy spectrum is caused by a cut-off of the light elements. The shape of the all-particle spectrum at higher energies is then determined by the subsequent cut-offs of all elemental species in cosmic rays. Most likely, the astrophysical origin of the knee is a combination of the maximum energy reached in the acceleration process and leakage from the Galaxy during propagation 19. More exotic ideas about the cause of the knee are most likely excluded 6 6 .

In conclusion this gives a qualitative 'standard picture' of the origin of galactic cosmic rays. However, several details remain unclear and a precise quantitative description of all aspects of the acceleration and propagation mechanisms is still missing. Among the open questions are:

It is not clear how to precisely match the spectral indices observed at Earth to the spectra at the sources, being compatible with the $\mathrm{TeV} \gamma$-ray observations and the modification of the spectral slope during propagation.

A precise astrophysical interpretation of air shower data is at present limited by the understanding of hadronic interactions in the atmosphere, thus the exact shape of the energy spectra at their corresponding knees is unknown.

Contemporary assumptions on the parameters of cosmic-ray propagation models yield anisotropies in the arrival directions not observed by air shower experiments 61[19].

In the near future experiments like TRACER aim to reveal details of the cosmic-ray propagation for energies approaching the knee [62]. Experiments at the LHC probing the extreme forward direction of phase space will improve the description of high-energy hadronic interactions 63. Also the exploration of the end of the galactic component and the transition to extragalactic particles in the energy range from $10^{17}-10^{18} \mathrm{eV}$ will be of importance. Key experiments in this energy region are KASCADE-Grande (a $0.5 \mathrm{~km}^{2}$ extension of the KASCADE experiment) 64, Ice Cube/ Ice Top (a $1 \mathrm{~km}^{2}$ air shower experiment and neutrino telescope at the South Pole) 6566, and HEAT/AMIGA (a $25 \mathrm{~km}^{2}$ extension of the Auger experiment to lower energies) 67.

Around $10^{18} \mathrm{eV}$ two features appear in the all-particle energy spectrum. The second knee at $E_{2 n d} \approx 400 \mathrm{PeV} \approx 92 \times E_{k}$, where the spectrum exhibits a steepening to $\gamma \approx-3.3$, and the ankle at about $4 \mathrm{EeV}$, above this energy the spectrum seems to flatten again to $\gamma \approx-2.7$. The region around $4 \mathrm{EeV}$ is sometimes also called "the dip" in the spectrum.

A possible cause for the second knee is the end of the galactic component, when all elements successively have reached their cut-off energies, the latter being proportional to their nuclear charge [4]19. If one assumes that ultra-heavy elements (heavier than iron) play an important role to understand the second knee, the factor of 92 between the energies of the knee and the second knee can be easily understood as the nuclear charge of the heaviest elements in the periodic table.

The dip is proposed to be caused by electronpositron pair production of cosmic rays on photons of the cosmic microwave background 68 .

The investigation of the transition region and a precise measurement of the galactic all-particle spectrum is also important for an estimate of the energy content of extragalactic cosmic rays. For example, the extragalactic component needed according to the poly-gonato model to sustain the observed all-particle flux at highest energies has an energy density of $\rho_{E}^{e x g}=3.7 \cdot 10^{-7} \mathrm{eV} / \mathrm{cm}^{3}$.

A promising rediscovered technique for the exploration of cosmic rays from the transition region to highest energies is the detection of radio signals from air showers 69. Most likely the emission mechanism is coherent synchrotron radiation of electrons with energies around the critical energy $(85 \mathrm{MeV})$ deflected in the magnetic field of the Earth (geosynchrotron radiation) 70. The LOPES experiment, registering showers in coincidence with the KASCADE-Grande experiment has demonstrated the feasibility of this approach 71]. Radio detection of air showers is also pursued in the CODALEMA experiment 72 and within the LOFAR radio telescope [73]. Also first radio pulses from air showers have been recorded with antennae set up at 
the southern site of the Pierre Auger Observatory 74.

\section{References}

[1] V. Hess, Phys. Zeitschr. 13 (1912) 1084.

[2] F. Halzen, astro-ph/0604441 (2006).

[3] T. Gaisser, Cosmic Rays and Particle Physics, Cambridge University Press, 1999.

[4] J. Hörandel, Astropart. Phys. 19 (2003) 193.

[5] J. Hörandel, Astropart. Phys. 21 (2004) 241.

[6] J. Hörandel, astro-ph/0702370 (Advances in Space Research in press) (2007).

[7] J. Hörandel, Il Nuovo Cimento B 120 (2005) 825.

[8] J. Hörandel, Int. J. Mod. Phys. A 20 (2005) 6753.

[9] M. Wiedenbeck, et al., Proc. 28th Int. Cosmic Ray Conf., Tsukuba 4 (2003) 1899.

[10] N. Yanasak, et al., Astrophys. J. 563 (2001) 768.

[11] M. Garcia-Munoz, et al., Astrophys. J. 201 (1975) L141.

[12] J. Simpson, M. Garcia-Munoz, Space Sci. Rev. 46 (1988) 205.

[13] M. Garzia-Munoz, et al., Astrophys. J. Suppl. Ser. 64 (1987) 269.

[14] P. Boyle, et al., Proc. 30th Int. Cosmic Ray Conf., Merida (2007) in press.

[15] W. Jones, Proc. 29th Int. Cosmic Ray Conf., Pune 10 (2005) 173.

[16] T. Guzik, et al., Adv. Space Res. (2004) in press.

[17] E. Seo, et al., Adv. Space Res. 33 (2004) 1777.

[18] F. Gahbauer, et al., Astrophys. J. 607 (2004) 333.

[19] J. Hörandel, et al., Astropart. Phys. 27 (2007) 119.

[20] J. Matthews, Astropart. Phys. 22 (2005) 387.

[21] J. Hörandel, Mod. Phys. Lett. A 22 (2007) 1533.

[22] P. Doll, et al., Proc. 30th Int. Cosmic Ray Conf., Merida (2007) in press.

[23] J. Hörandel, J. Phys. G: Nucl. Part. Phys. 29 (2003) 2439.

[24] H. Ulrich, et al., Proc. 30th Int. Cosmic Ray Conf., Merida (2007) in press.

[25] T. Antoni, et al., Nucl. Instr. \& Meth. A 513 (2003) 490.

[26] J. Engler, et al., Nucl. Instr. \& Meth. A 427 (1999) 528.

[27] P. Doll, et al., Nucl. Instr. \& Meth. A 488 (2002) 517.

[28] T. Antoni, et al., J. Phys. G: Nucl. Part. Phys. 25 (1999) 2161.

[29] T. Antoni, et al., J. Phys. G: Nucl. Part. Phys. 27 (2001) 1785.

[30] J. Milke, et al., Acta Physica Polonica B 35 (2004) 341.

[31] J. Milke, et al., Proc. 29th Int. Cosmic Ray Conf., Pune 6 (2005) 125.

[32] W. Apel, et al., J. Phys. G: Nucl. Part. Phys. (2007) in press.

[33] J. Hörandel, et al., Proc. 29th Int. Cosmic Ray Conf., Pune 6 (2005) 121.

[34] J. Hörandel, Nucl. Phys. B (Proc. Suppl.) 122 (2003) 455.

[35] T. Antoni, et al., Astropart. Phys. 24 (2005) 1.
[36] G. Navarra, et al., Proc. 28th Int. Cosmic Ray Conf., Tsukuba 1 (2003) 147.

[37] Y. Hayashi, et al., Proc. 29th Int. Cosmic Ray Conf., Pune 10 (2005) 243.

[38] M. Amenomori, et al., Phys. Rev. D 62 (2000) 112002.

[39] S. Over, et al., Proc. 30th Int. Cosmic Ray Conf., Merida (2007) in press.

[40] T. Antoni, et al., Astrophys. J. 604 (2004) 687.

[41] G. Maier, et al., Int. J. Mod. Phys. A 20 (2005) 6840.

[42] J. Candia, et al., J. Cosmol. Astropart. Phys. 5 (2003) 3.

[43] F. Aharonian, et al., Astron. \& Astroph. 449 (2006) 223.

[44] J. Hinton, New Astronomy Reviews 48 (2004) 331.

[45] D. Ferenc, et al., Nucl. Instr. \& Meth. A 553 (2005) 274.

[46] T. Weekes, et al., Astropart. Phys. 17 (2002) 221.

[47] W. Baade, F. Zwicky, Phys. Rev. 46 (1934) 76.

[48] E. Fermi, Phys. Rev. 75 (1949) 1169.

[49] W. Axford, et al., Proc. 15th Int. Cosmic Ray Conf., Plovdiv 11 (1977) 132.

[50] G. Krymsky, Dok. Acad. Nauk USSR 234 (1977) 1306.

[51] A. Bell, Mon. Not. R. Astr. Soc. 182 (1978) 147.

[52] R. Blanford, J. Ostriker, Astrophys. J. 221 (1978) L29.

[53] F. Aharonian, et al., Nature 432 (2004) 75.

[54] H. Völk, E. Berezhko, Astron. \& Astroph. 451 (2006) 981.

[55] E. Berezhko, H. Völk, Astrophys. J. 661 (2007) L175.

[56] F. Aharonian, et al., Astron. \& Astroph. 370 (2001) 112.

[57] E. Berezhko, et al., Astron. \& Astroph. 400 (2003) 971.

[58] H. Völk, astro-ph/0603502 (2006).

[59] A. Strong, J. Mattox, Astron. \& Astroph. 308 (1996) L21.

[60] A. Strong, I. Moskalenko, preprint astro-ph/9903370 (1999).

[61] V. Ptuskin, Adv. Space Res. 19 (1997) 697.

[62] D. Müller, et al., Proc. 30th Int. Cosmic Ray Conf., Merida (2007) in press.

[63] R. Engel, Nucl. Phys. B (Proc. Suppl.) 151 (2006) 437.

[64] G. Navarra, et al., Nucl. Instr. \& Meth. A 518 (2004) 207.

[65] M. Kestel, et al., Nucl. Instr. \& Meth. A 535 (2004) 139.

[66] T. Gaisser, et al., Proc. 28th Int. Cosmic Ray Conf., Tsukuba 2 (2003) 1117.

[67] H. Kalges, et al., Proc. 30th Int. Cosmic Ray Conf., Merida (2007) in press.

[68] V. Berezinsky, astro-ph/0509069 (2005).

[69] H. Allan, Progress in Elementary Particles and Cosmic Ray Physics, J.G. Wilson \& S.G. Wouthuysen eds., North Holland, 1971, p. 169.

[70] T. Huege, H. Falcke, Astropart. Phys. 24 (2005) 116.

[71] H. Falcke, et al., Nature 435 (2005) 313.

[72] D. Ardouin, et al., Nucl. Instr. \& Meth. A 555 (2005) 148.

[73] www.lofar.org.

[74] A. van den Berg, et al., Proc. 30th Int. Cosmic Ray Conf., Merida (2007) in press. 